\title{
Adverse orienting effects on visual working memory encoding and maintenance
}

\author{
Benchi Wang ${ }^{1}$ Chuyao Yan $^{2}$ - Zhiguo Wang ${ }^{2,3}$ - Christian N. L. Olivers ${ }^{1}$. \\ Jan Theeuwes ${ }^{1}$
}

Published online: 28 November 2016

(C) The Author(s) 2016. This article is published with open access at Springerlink.com

\begin{abstract}
Visual working memory (VWM) representations can be strengthened by pre-cues presented before, and retrocues presented after, the memory display, providing evidence that attentional orienting plays a role in memory encoding and maintenance. It is unknown whether attentional orienting to VWM stimuli can also have adverse effects (known as inhibition of return; IOR), as has been found for perceptual-cueing tasks. If so, this would provide further evidence for common attentional orienting mechanisms for mnemonic and perceptual representations. In Experiment 1, we used pre-cueing and demonstrated an increased encoding probability, but not precision, at short SOAs, but probability decreased at long SOAs, reminiscent of the classic IOR findings. In Experiment 2, we used retro-cueing and showed that it improved memory performance, unless attention was cued back to the center of the display by a second cue. In this case, the deleterious effects were on precision, indicating that the item was still retained, but its quality of representation suffered. Together, these results provide further evidence for universal spatial attentional
\end{abstract}

Electronic supplementary material The online version of this article (doi:10.3758/s13423-016-1205-4) contains supplementary material, which is available to authorized users.

Benchi Wang

wangbenchi.swift@gmail.com

1 Department of Experimental and Applied Psychology, Vrije Universiteit Amsterdam, Van der Boechorststraat 1, 1081 BT Amsterdam, The Netherlands

2 Department of Psychology, Hangzhou Normal University, Hangzhou, China

3 Department of Cognitive Science, Macquarie University, Sydney, Australia mechanisms operating on perceptual as well as mnemonic representations.

Keywords Visual working memory · Inhibition of return · Pre-cue $\cdot$ Retro-cue

Visual working memory (VWM) is a limited-capacity system that serves to temporally maintain visual information and is essential for many ongoing cognitive tasks. The role of attention in VWM is undisputed: It affects encoding into VWM (Schmidt, Vogel, Woodman, \& Luck, 2002), is used to retrieve information from VWM (Theeuwes, Kramer, \& Irwin, 2011), and plays a crucial role in the selective maintenance of items within VWM (Vogel, Woodman, \& Luck, 2005). There are several ways to orient attention to specific items in memory. For example, when a pre-cue is presented before the memory array, attention focuses onto its spatial location, and items presented at that location are more likely to be transferred into VWM (Awh \& Pashler, 2000; Makovski \& Jiang, 2007; Schmidt et al., 2002; Vogel et al., 2005). Alternatively, cues can be presented after the memory array has been switched off, allowing the orientation of attention to a spatial location within an internal memory representation (Delvenne, Cleeremans, \& Laloyaux, 2009; Griffin \& Nobre, 2003; Makovski \& Jiang, 2007; Murray, Nobre, Clark, Cravo, \& Stokes, 2013; van Moorselaar, Gunseli, Theeuwes, \& Olivers, 2015; van Moorselaar, Olivers, Theeuwes, Lamme, \& Sligte, 2015). Such so-called retro-cues improve the memory representation of the cued item.

In perceptual attention research, there is also a long research tradition investigating adverse effects of attentiondirecting cues on perceptual processing (Klein, 2000; Posner \& Cohen, 1984; Wang, Hilchey, Cao, \& Wang, 2014). This type of research uses a typical task, in which a cue appears to 
either the left or the right of fixation. Typically, participants are faster and more accurate in responding to targets that appear at the cued than at the uncued location, referred to as the cue facilitation effect (Posner, 1980). Notably, when there is a delay between the offset of the cue and the onset of the target, participants are slower in responding to targets at the cued than at the uncued location, an effect called inhibition of return (IOR; cf. Posner \& Cohen, 1984). Thus, orienting in the outside world shows a typical biphasic pattern in which attentional facilitation is followed by inhibition. It has been argued that the mechanism responsible for IOR serves a novelty-seeking, foraging function by inhibiting attention from returning to previously examined locations (Klein, 2000).

Previous studies have shown that IOR is mediated by spatial working memory (Castel, Pratt, \& Craik, 2003). Here we report two experiments investigating the reverse: the extents to which IOR modulates VWM encoding (Exp. 1, using a precueing procedure) and maintenance (Exp. 2, using a retrocueing procedure).

\section{Experiment 1}

In Experiment 1, we tested whether IOR-like effects occurred on encoding into VWM. We first presented a spatial cue that was either valid or invalid. The SOA between the cue and the memory display was either short (200 ms) or long (400 ms). Previous studies investigating IOR on perceptual processing have shown that with these SOAs, one should observe attentional facilitation followed by inhibition (see Klein, 2000, for a review). We tested whether the same biphasic effect seen in perceptual processing can be found on VWM performance. More specifically, we investigated whether IOR affected the probability of encoding, or the precision, by employing a mixture-modeling approach (Bays, Catalao, \& Husain, 2009).

\section{Method}

Participants Sixteen adults ( 11 females, 5 males; mean age $=22.8$ years) took part for money compensation or course credits. They all provided written informed consent, and all reported normal color vision and normal or corrected-to-

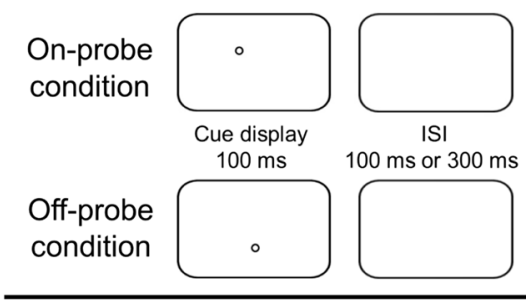

Fig. 1 The procedure of Experiment 1. A pre-cue was shown for $100 \mathrm{~ms}$ before the memory display (300 ms), and the interstimuli interval (ISI) could be 100 and $300 \mathrm{~ms}$. After a delay period $(1,000 \mathrm{~ms})$, a color wheel normal visual acuity. Eight participants were run in China, and eight were run in the Netherlands.

Apparatus An HP Compaq 8000 Elite computer with a 21-in. color monitor controlled the timing of the events and generated stimuli on a gray screen $\left(17 \mathrm{~cd} / \mathrm{m}^{2}\right.$ in China, and $19 \mathrm{~cd} / \mathrm{m}^{2}$ in the Netherlands). A CRT monitor was used in China, and an LCD monitor was used in the Netherlands. Stimulus presentation and response registration were controlled by custom scripts written in Python. Participants were tested in a dimly lit laboratory, and they held their head on a chinrest located $71 \mathrm{~cm}$ away from the monitor. The research protocol was approved by the Scientific and Ethical Review Committee of the Faculty of Psychology and Education of VU University and by the Institutional Review Board of the Center for Cognition and Brain Disorders, Hangzhou Normal University.

Stimuli Participants were required to memorize the colors of five colored squares $\left(0.6^{\circ} \times 0.6^{\circ}\right)$. For each trial, the color of each square was randomly chosen from one of 12 equiluminant colors (ranges: $65-70 \mathrm{~cd} / \mathrm{m}^{2}$ in China and 36$44 \mathrm{~cd} / \mathrm{m}^{2}$ in the Netherlands), evenly distributed along a color circle in the CIE $L * a * b *$ color space (centered at $L=70, a=5$, $\mathrm{b}=0$, with a radius of 60 ), without replacement, and their locations were randomly chosen from eight equally spaced positions along an imaginary ring with a radius of $3^{\circ}$ (see Fig. 1).

Procedure and design A small fixation point $\left(0.67^{\circ} \times 0.67^{\circ}\right)$ was presented throughout the trial. A white circle serving as a pre-cue $\left(217 \mathrm{~cd} / \mathrm{m}^{2}\right.$ in China and $176 \mathrm{~cd} / \mathrm{m}^{2}$ in the Netherlands) with a radius of $0.5^{\circ}$ was presented for $100 \mathrm{~ms}$. This cue appeared at the probed item's location (on-probe condition) or at one of the nonprobed items' locations (offprobe condition), followed by a time interval of either 100 or $300 \mathrm{~ms}$, randomized within blocks. Then the memory array was presented for $300 \mathrm{~ms}$. After a 1,000-ms memory delay, the probe display was presented until response, containing five empty squares $\left(0.6^{\circ} \times 0.6^{\circ}\right)$ and a continuous response wheel (subtending $1.5^{\circ}$ wide, $7^{\circ}$ radius) of 180 color segments. This display remained on until the response. A bolder square indicated which item participants had to report by

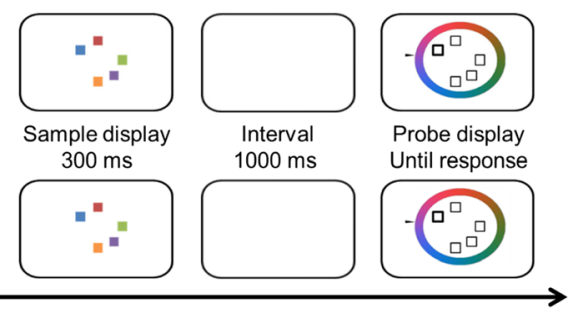

representing continuous color values and a test cue signaling the to-berecalled item were presented. Participants recalled the feature value of the indicated item by using a mouse to select a value on the wheel 
selecting one of 180 values on the color wheel (randomly rotated), using the mouse. The selected color value was indicated by a small black arrowhead cursor. While the mouse was being moved, the cued square was filled with the color value matching the selected value. Accuracy was emphasized. There were 2 (Pre-Cue Location: on-probe vs. off-probe) $\times 2$ (SOA: 200 vs. $400 \mathrm{~ms}$ ) conditions, randomly mixed, run in two successive sessions held within 5 days of each other. Each condition contained 200 trials, for a total of 800 trials. The experiment was preceded by 20 practice trials. To maximize the number of trials for each condition, the validity of the precue was $50 \%$, although this might in fact be suboptimal for observing inhibition.

Analysis Response error was calculated by subtracting each probed item's correct value from the response value. A mixture-modeling analysis (Bays, Catalao, \& Husain, 2009) was conducted on these response error data (see the supplementary material, Figs. S1 and S2, for a histogram of the response error data across all participants), to derive the parameters of the response error distribution. This distribution was assumed to consist of a uniform distribution of response errors (for guessing trials), one von Mises (circular normal) distribution of response errors for nonguessing trials, and four von Mises distributions of response errors for memory substitutions, in which observer reported one of the other items in the display. By using maximum likelihood estimation, the distribution of the response error data from each condition was entered into the model $p(e)=(1-g-\beta) \phi_{\sigma}(e)+g \frac{1}{2 \pi}+\beta \frac{1}{m} \sum_{1}^{m} \phi_{\sigma}\left(e_{m}\right)$

where two input parameters $e$ (response errors) and $m$ (the number of nontarget items) are required, and three output parameters $g$ (guess rate; i.e., the proportion of the guess trials), $\beta$ (swap errors; i.e., the proportion of misremembering trials), and $\sigma$ (standard deviation; i.e., the width of the mixture distribution, reflecting the precision of the memory representation) will be given. We used the MemToolbox (a MATLAB toolbox; Suchow, Brady, Fougnie, \& Alvarez, 2013) to fit the mixture distribution of the response errors. We also fitted the two-parameter model of Zhang and Luck (2008), which yielded the same pattern of results (see the supplementary material).

\section{Results}

The response error distribution and its model-fitted results (see the supplementary material, Fig. S1) for each condition were derived from the best-fitting parameter values of the overall trials. The guess rate $(g)$, swap error $(\beta)$, and standard deviation $(\sigma)$ were each entered in a repeated measures analysis of variance (ANOVA) with Pre-Cue Location (on-probe vs. offprobe) and SOA (200 vs. $400 \mathrm{~ms}$ ) as factors.

Figure 2a (left panel) presents the means of the guess rates for all conditions, and Fig. $2 \mathrm{~b}$ (left panel) presents the differences in guess rates between the different pre-cue locations. No significant main effect was observed for pre-cue location, $F<1, p>.9$, although there was a tendency toward fewer

\section{A) Memory performance}
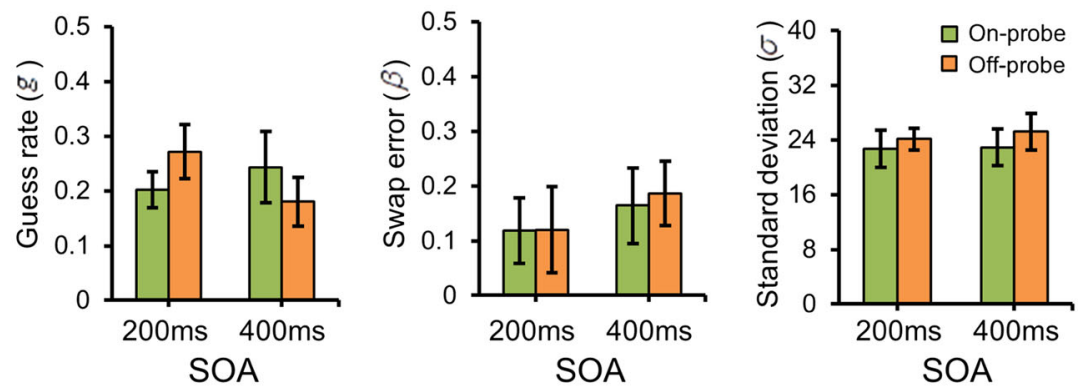

\section{B) Difference between on/off probe}
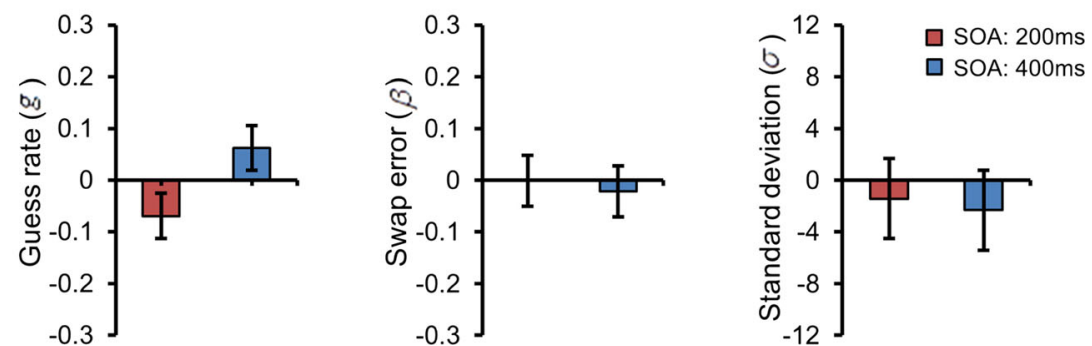

Fig. 2 The results of Experiment 1. (a) Guess rates, swap errors, and standard deviations of each condition. (b) Differences between the on-and offprobe conditions in terms of guess rates, swap errors, and standard deviations. Error bars denote within-subjects 95\% confidence intervals (Morey, 2008) 
guesses with increasing SOA, though this was not significant, $F(1,15)=3.1, p=.1, \eta_{\mathrm{p}}{ }^{2}=.17$. Importantly, however, we observed a significant interaction, $F(1,15)=18.5, p=.001$, $\eta_{\mathrm{p}}{ }^{2}=.55$. Planned comparisons showed that at SOA $200 \mathrm{~ms}$, the guess rate was marginally lower for on- than for off-probe conditions, $t(15)=1.89, p=.079$, an effect that was reversed in the SOA 400-ms condition, in which the guess rate was now higher for on- than for off-probe performance, $t(15)=2.4, p=.03$.

The middle and right panels of Fig. 2a present the means of swap errors and standard deviations, respectively, for all conditions, and the same panels in Fig. 2 b present the differences in swap errors and standard deviations, respectively, between the different pre-cue locations. No significant main effects or interactions were observed for either of these measures, all $F \mathrm{~s}$ $<2.1$, all $p s>.17$.

\section{Discussion}

The present experiment shows the classic biphasic effect of pre-cues that is reminiscent of IOR, but now on VWM performance, with early facilitation at the short SOA followed by inhibition at the long SOA. Furthermore, this effect was found for memory probability (reflected by the guess rate), but not for memory precision (reflected by the standard deviation). We propose that at the long SOA, due to IOR, the (pre-cued) location became inhibited before the sample display was presented. Once the display was presented, IOR at that location reduced the probability that the stimulus presented at that location would be encoded into VWM. However, if it was encoded, it was represented with the same precision as when it was attended. This suggests an all-or-none mechanism of IOR, in which on some trials attention was still there (with full precision as a consequence), whereas on other trials it was withdrawn (and the item was not even encoded).

\section{Experiment 2}

In Experiment 2, we employed retro-cuing in which the cue was presented after extinguishing the memory array. Following the presentation of a retro-cue, it is likely that attention remains focused on the location of the retro-cue unless we force attention to move elsewhere. To reorient attention away from the cued location, we used a cued-back condition (as was used in the classic Posner \& Cohen [1984] IOR paradigm) to promote the reorientation of attention away from the cued item, and thereby inhibit the return of attention to that item. In Experiment 2, in one condition, we presented a second cue in the center of the display to draw attention back to the center. Because the mechanisms underlying IOR bias responses against previously attended locations, the location of the retro-cue should become suppressed when attention is drawn back to the center.

\section{Method}

Thirty-one adults (18 females, 13 males; mean age 23.9 years) took part for money compensation or course credits. The procedure was similar to that of Experiment 1, except that instead of a pre-cue, a retro-cue was presented $200 \mathrm{~ms}$ after the offset of the memory display. The retro-cue was shown for $100 \mathrm{~ms}$, followed by a random retention interval ( $350-450 \mathrm{~ms})$. Then, depending on the condition, either no second cue was presented (not-cued-back condition) or a second cue was presented for $300 \mathrm{~ms}$ at the center of the screen (cued-back condition); see Fig. 3 for an example. Each participant completed 20 practice trials and 1,000 experimental trials, with 2 (RetroCue Location: on-probe vs. off-probe) $\times 2$ (Attention Shift: cued back vs. not cued back) conditions randomly mixed. The validity of the retro-cue (i.e., the proportion of the on-probe condition) was $20 \%$, to make sure each to-be-memorized item had an equally cued probability, so that each on-probe condition contained 100 trials and each off-probe condition contained 400 trials. In all other respects, including the data analysis, this experiment was the same as Experiment 1.

\section{Results}

The response error distribution and its model-fitted results for each condition are illustrated in the supplementary material, Fig. S2. As in Experiment 1, repeated measures ANOVAs were conducted on the guess rates $(g)$, swap errors $(\beta)$, and

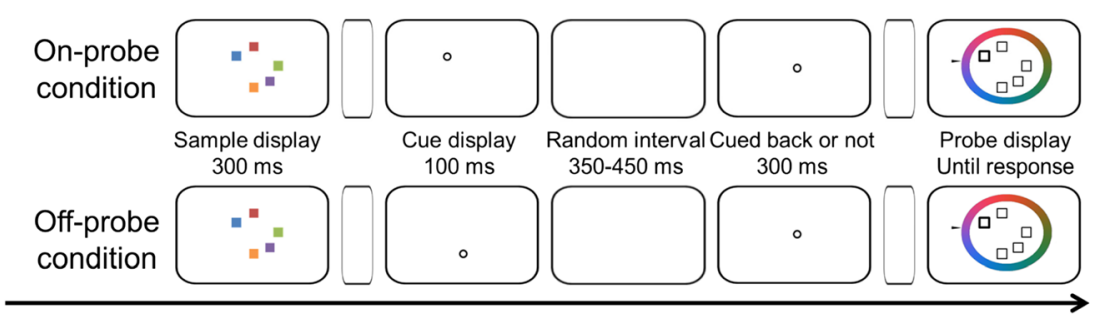

Fig. 3 The procedure of Experiment 2. First, the memory display was shown for $300 \mathrm{~ms}$, and then the retro-cue was shown for $100 \mathrm{~ms}$, after an interval of $200 \mathrm{~ms}$. A random interval $(350-450 \mathrm{~ms})$ followed the retrocue, and then another cue or no cue. Finally, a color wheel representing continuous color values and a test cue signaling the to-be-recalled item were presented. Participants recalled the feature value of this item by using a mouse to select a value on the wheel 
standard deviations $(\sigma)$, separately, with the variables RetroCue Location (on-probe vs. off-probe) and Attentional Shift (cued back vs. not cued back).

The means of the guess rates for all conditions are presented in Fig. 4a (left panel), and the differences in guess rates between the on-probe and off-probe conditions are presented in Fig. 4b (left panel). We found a main effect of retro-cue location, $F(1$, $30)=8.94, p=.006, \eta_{\mathrm{p}}{ }^{2}=.23$, but not of attention shift, $F(1$, $30)=2.04, p=.163, \eta_{\mathrm{p}}{ }^{2}=.06$. There was no two-way interaction, $F(1,30)=0.001, p=.979, \eta_{\mathrm{p}}^{2}<.001$. These data indicate a reliable retro-cue benefit, since the guess rate was lower in the on-probe than in the off-probe condition, no matter whether or not attention was shifted to the center.

Figure 4a (middle panel) shows the mean swap errors for all conditions, and the differences in swap errors between the on-probe and off-probe conditions are shown in Fig. 4b (middle panel). No significant main effects or interactions were observed, all $F_{\mathrm{s}}<2.4$, all $p \mathrm{~s}>.132$.

Finally, Fig. 4a (right panel) shows the means of the standard deviations for all conditions. Figure $4 \mathrm{~b}$ (right panel) shows the differences in standard deviations between the on-probe and off-probe conditions. We observed a significant main effect of attention shift, $F(1,30)=6.37, p=.015, \eta_{\mathrm{p}}{ }^{2}=.18$, but not of retro-cue location (on- and off-probe condition), $F(1,30)=$ $0.16, p=.688, \eta_{\mathrm{p}}{ }^{2}=.01$. Importantly, there was a reliable two-way interaction, $F(1,30)=9.44, p=.004, \eta_{\mathrm{p}}{ }^{2}=.24$. Planned comparisons indicated that in the on-probe condition, the standard deviation was lower than in the off-probe condition when there was no cue back, $t(30)=2.46, p=.02$, but that this decreased standard deviation was no longer present when the cue back was delivered, $t(30)=1.38, p=.178$.

\section{Discussion}

Consistent with previous studies, a clear retro-cue benefit was observed for both memory probability (in terms of guess rate) and memory precision (in terms of standard deviation). When the retro-cue was valid, the memory probability of the cued item was significantly increased in both the cued-back condition and the not-cued-back condition, showing that attention was directed to the cued item, at the expense of the noncued items. More importantly, although the benefit for probability was retained, the retro-cue benefit for memory precision was no longer present in the cued-back condition relative to the condition in which there was no second cue (the not-cuedback condition), resulting in a cue by probe location interaction. Thus, memory precision was relatively suppressed, consistent with a IOR-like mechanism.

Note that in the cued-back condition, the on-probe location was no worse than the off-probe location. One may argue if this on-probe item had been inhibited (as a mechanism such as IOR would imply) because attention was pulled away by the second cue, one would have expected worse performance in this condition (the on-probe cued-back condition) than in the off-probe (cued-back) condition. However we argue that memory, unlike attention, serves to create a sustained representation. By cueing an item, it is consolidated for later report, as is evident from the fact that probability of report improved reliably for cued items - regardless of whether or not attention was then cued away again. This means that any inhibitory attention-related effects can only start to operate after - and thus will be expressed relative to-a sustained benefit. Interestingly, our data are consistent with such mechanisms:

\section{A) Memory performance}
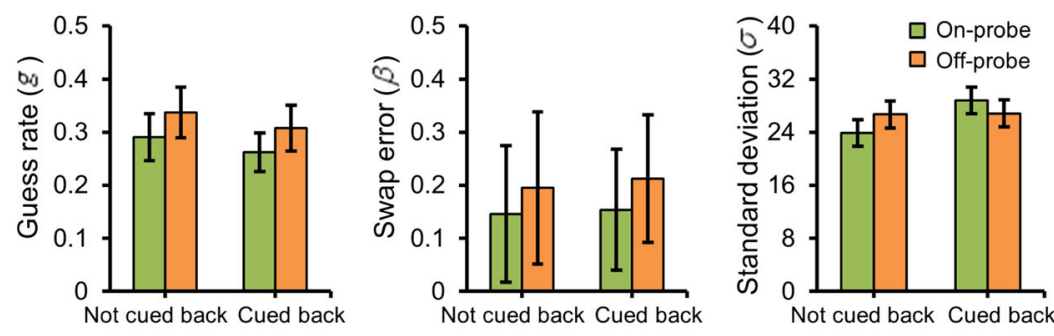

\section{B) Difference between on/off probe}
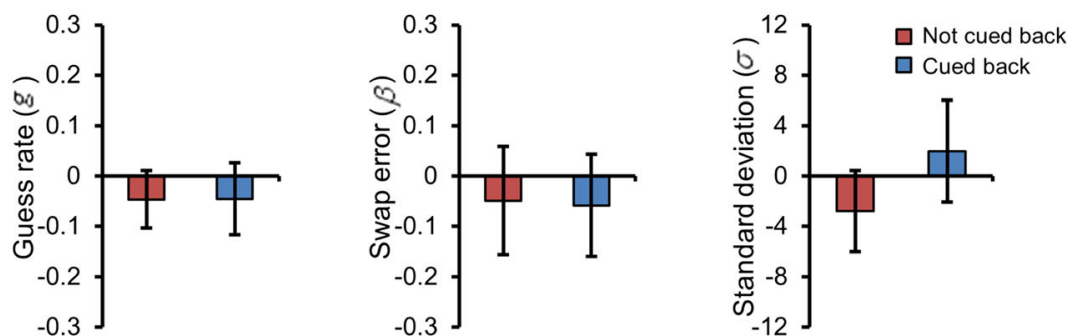

Fig. 4 The results of Experiment 2. (a) Guess rates, swap errors, and standard deviations of each condition. (b) Differences between the on- and offprobe conditions in terms of guess rates, swap errors, and standard deviations. Error bars denote within-subjects $95 \%$ confidence intervals 
We did not see an effect of the second cue on encoding probability (because the first cue ensured that the item was already consolidated in memory, even when attention was later cued back), but instead an effect on memory precision. This dissociation between probability and precision also explains why we came to a different conclusion than Hollingworth and Maxcey-Richard (2013), who claimed that VWM maintenance should be dissociated from the locus of visual attention. In their experiment, they found that attention shifts during the delay period had no impact on retro-cue benefits. However, they did not include memory precision as a measure, only the probability of correct report. As we showed here, cueing attention away during the delay period may indeed not affect the probability of reporting an item, only its precision.

\section{General discussion}

The present study reveals adverse mechanisms of spatial orienting on VWM performance that are reminiscent of IOR observed for perceptual presentations. In Experiment 1 we used a pre-cue to orient attention to specific items in the memory array and revealed a biphasic effect on the probability of encoding the item into VWM, with first facilitation and then inhibition. Previous studies have shown a facilitation effect of pre-cues on memory performance (e.g., Awh \& Pashler, 2000); the present study is the first to show that such cues also have adverse effects on VWM performance. For Experiment 2 we used a retro-cue technique, and consistent with previous studies, memory performance benefits (memory probability in the present study) appeared when the item that was retro-cued needed to be recalled (on-probe) versus the condition in which another item needed to be recalled (off-probe; see, e.g., Griffin $\&$ Nobre, 2003). Crucially, however, when attention was pulled back to the middle of the screen, the retro-cue benefit on memory precision waned, whereas the benefits on probability remained. This suggests a suppression of memory precision when attention was withdrawn, an effect similar to the occurrence of IOR in Posner-like attentional-cueing tasks.

The present findings are consistent with a large functional overlap between attentional and VWM mechanisms (Awh \& Jonides, 2001; Chun, 2011; Kiyonaga \& Egner, 2012, 2016; Olivers, 2008; Peters, Kaiser, Rahm, \& Bledowski, 2015; Sahan, Verguts, Boehler, Pourtois, \& Fias, 2015; Theeuwes, Belopolsky, \& Olivers, 2009). Although IOR is functional when searching for an object in the outside world (facilitation of foraging; Klein, 2000), it may be less useful when holding items in VWM. In addition to the role of IOR as foraging facilitator in visual search, recent studies have stressed the effect of IOR on motoric/decision-making processes (so-called output-based IOR; see Hilchey, Klein, \& Satel, 2014), especially when participants make saccadic eye movements. Even though we did not monitor eye movements, in the present study it is highly unlikely that eye movements were made, because participants were instructed to memorize the colors of five squares presented on an imaginary circle around fixation. If anything, the best strategy to memorize the colors was to remain fixated on the center. Furthermore, it is unclear how motor inhibition would explain a reduced probability of encoding (Exp. 1) or memory precision (Exp. 2).

The present study shows that pre-cues led to reduced memory probability (at a long SOA), whereas retro-cues led to reduced memory precision (when attention was withdrawn). It is likely that this difference relates to a fundamental difference in the information that was available at the time of cueing. At the moment of a pre-cue, by definition, no item information is yet available, only location information. Attending to that location will thus help reduce the effective set size (at the expense of the other items) and increase the likelihood of encoding the cued item. In the retro-cue condition, however, the item has already been encoded (at least with high likelihood). Given this benefit, there is no incentive to drop it. All that can then be affected by an inhibitory mechanism is the precision of the memory. Thus, we suggest that the attentional mechanisms of pre- and retro-cueing may be the same, including IOR, but that the effects may differ, depending on whether the to-be-remembered item is already available or still has to be encoded.

Author note This research was supported by an European Research Council (ERC) advanced grant [ERC-2012-AdG-323413] to J.T., an ERC consolidator grant [ERC-2013-CoG-615423] to C.O., a National Natural Science Foundation of China grant (31371133) to Z.W., and a China Scholarship Council scholarship (201508330313) to B.W.

Open Access This article is distributed under the terms of the Creative Commons Attribution 4.0 International License (http:// creativecommons.org/licenses/by/4.0/), which permits unrestricted use, distribution, and reproduction in any medium, provided you give appropriate credit to the original author(s) and the source, provide a link to the Creative Commons license, and indicate if changes were made.

\section{References}

Awh, E., \& Jonides, J. (2001). Overlapping mechanisms of attention and spatial working memory. Trends in Cognitive Sciences, 5, 119-126. doi:10.1016/S1364-6613(00)01593-X

Awh, E., \& Pashler, H. (2000). Evidence for split attentional foci. Journal of Experimental Psychology: Human Perception and Performance, 26, 834-846. doi:10.1037/0096-1523.26.2.834

Bays, P. M., Catalao, R. F. G., \& Husain, M. (2009). The precision of visual working memory is set by allocation of a shared resource. Journal of Vision, 9(10), 7. doi:10.1167/9.10.7

Castel, A. D., Pratt, J., \& Craik, F. I. M. (2003). The role of spatial working memory in inhibition of return: Evidence from divided 
attention tasks. Perception \& Psychophysics, 65, 970-981. doi:10.3758/BF03194827

Chun, M. M. (2011). Visual working memory as visual attention sustained internally over time. Neuropsychologia, 49, 1407-1409. doi:10.1016/j.neuropsychologia.2011.01.029

Delvenne, J.-F., Cleeremans, A., \& Laloyaux, C. (2009). Feature bindings are maintained in visual short-term memory without sustained focused attention. Experimental Psychology, 57, 108-116. doi:10.1027/1618-3169/a000014

Griffin, I. C., \& Nobre, A. C. (2003). Orienting attention to locations in internal representations. Journal of Cognitive Neuroscience, 15, 1176-1194. doi:10.1162/089892903322598139

Hilchey, M. D., Klein, R. M., \& Satel, J. (2014). Returning to "inhibition of return" by dissociating long-term oculomotor IOR from short-term sensory adaptation and other nonoculomotor "inhibitory" cueing effects. Journal of Experimental Psychology: Human Perception and Performance, 40, 1603-1616. doi:10.1037/a0036859

Hollingworth, A., \& Maxcey-Richard, A. M. (2013). Selective maintenance in visual working memory does not require sustained visual attention. Journal of Experimental Psychology: Human Perception and Performance, 39, 1047-1058. doi:10.1037/a0030238

Kiyonaga, A., \& Egner, T. (2012). Working memory as internal attention: Toward an integrative account of internal and external selection processes. Psychonomic Bulletin \& Review, 20, 228-242. doi:10.3758/s13423-012-0359-y

Kiyonaga, A., \& Egner, T. (2016). Center-surround inhibition in working memory. Current Biology, 26, 64-68. doi:10.1016/j. cub.2015.11.013

Klein, R. (2000). Inhibition of return. Trends in Cognitive Sciences, 4, 138-147. doi:10.1016/S1364-6613(00)01452-2

Makovski, T., \& Jiang, Y. V. (2007). Distributing versus focusing attention in visual short-term memory. Psychonomic Bulletin \& Review, 14, 1072-1078. doi:10.3758/BF03193093

Morey, R. D. (2008). Confidence intervals from normalized data: A correction to Cousineau (2005). Tutorial in Quantitative Methods for Psychology, 4, 61-64. doi:10.3758/s13414-012-0291-2

Murray, A. M., Nobre, A. C., Clark, I. A., Cravo, A. M., \& Stokes, M. G. (2013). Attention restores discrete items to visual short-term memory. Psychological Science, 24, 550-556. doi:10.1177/0956797612457782

Olivers, C. N. L. (2008). Interactions between visual working memory and visual attention. Frontiers in Bioscience, 13, 1182-1191.

Peters, B., Kaiser, J., Rahm, B., \& Bledowski, C. (2015). Activity in human visual and parietal cortex reveals object-based attention in working memory. Journal of Neuroscience, 35, 3360-3369. doi:10.1523/JNEUROSCI.3795-14.2015

Posner, M. I. (1980). Orienting of attention. Quarterly Journal of Experimental Psychology, 32, 3-25. doi:10.1080/00335558008248231

Posner, M. I., \& Cohen, Y. (1984). Components of visual orienting. In H. Bouma \& D. G. Bouwhuis (Eds.), Attention and performance X: Control of language processes (pp. 531-556). Hillsdale, NJ: Erlbaum.

Sahan, M. I., Verguts, T., Boehler, C. N., Pourtois, G., \& Fias, W. (2015). Paying attention to working memory: Similarities in the spatial distribution of attention in mental and physical space. Psychonomic Bulletin \& Review, 23, 1190-1197. doi:10.3758/s13423-015-0990-5

Schmidt, B. K., Vogel, E. K., Woodman, G. F., \& Luck, S. J. (2002). Voluntary and automatic attentional control of visual working memory. Perception \& Psychophysics, 64, 754-763. doi:10.3758/BF03194742

Suchow, J. W., Brady, T. F., Fougnie, D., \& Alvarez, G. A. (2013). Modeling visual working memory with the MemToolbox. Journal of Vision, 13(10), 9. doi:10.1167/13.10.9

Theeuwes, J., Belopolsky, A., \& Olivers, C. N. L. (2009). Interactions between working memory, attention and eye movements. Acta Psychologica, 132, 106-114. doi:10.1016/j.actpsy.2009.01.005

Theeuwes, J., Kramer, A. F., \& Irwin, D. E. (2011). Attention on our mind: The role of spatial attention in visual working memory. Acta Psychologica, 137, 248-251. doi:10.1016/j.actpsy.2010.06.011

van Moorselaar, D., Gunseli, E., Theeuwes, J., \& Olivers, C. N. L. (2015). The time course of protecting a visual memory representation from perceptual interference. Frontiers in Human Neuroscience, 8, 1053. doi:10.3389/fnhum.2014.01053

van Moorselaar, D., Olivers, C. N. L., Theeuwes, J., Lamme, V. A. F., \& Sligte, I. G. (2015). Forgotten but not gone: Retro-cue costs and benefits in a double-cueing paradigm suggest multiple states in visual short-term memory. Journal of Experimental Psychology: Learning, Memory, and Cognition, 41, 1755-1763. doi:10.1037/xlm0000124

Vogel, E. K., Woodman, G. F., \& Luck, S. J. (2005). Pushing around the locus of selection: Evidence for the flexibleselection hypothesis. Journal of Cognitive Neuroscience, 17, 1907-1922. doi:10.1162/089892905775008599

Wang, B., Hilchey, M. D., Cao, X., \& Wang, Z. (2014). The spatial distribution of inhibition of return revisited: No difference found between manual and saccadic responses. Neuroscience Letters, 578, 128-132. doi:10.1016/j.neulet.2014.06.050

Zhang, W., \& Luck, S. J. (2008). Discrete fixed-resolution representations in visual working memory. Nature, 453, 233-235. doi: 10.1038 /nature 06860 